\title{
Particularities of a manic episode with psychotic symptoms on the background of psycho-trauma in adolescents
}

\author{
Bianca-Georgiana Secrieru, Elena-Rodica Popescu, \\ Gabriela-Carmen Lupușoru, Roxana Chiriță
}

Bianca-Georgiana Secrieru - M.D., junior psychiatrist, Socola Institute of Psychiatry, Iași Elena-Rodica Popescu - M.D., PhD Student, assistant professor Grigore T. Popa University of Medicine and Pharmacy, Iași; Psychiatrist, Socola Institute of Psychiatry, Iași

Gabriela-Carmen Lupuşoru - M.D., Senior Infantile Neuropsychiatrist, Socola Institute of Psychiatry, Iași

Roxana Chiriță - M.D., PhD, Professor of Psyhchiatry Grigore T. Popa University of Medicine and Pharmacy, Iași, Senior psychiatrist Socola Institute of Psychiatry, Iași

\begin{abstract}
Background: Psychosis is a common and functionally disruptive symptom of many psychiatric, neurodevelopmental, neurologic, and medical conditions and an important target of evaluation and treatment in neurologic and psychiatric practice. Objective: The purpose of this paper is to outline the importance of the evolution of depression, psychosis and mania among adolescents, and to provide a general perspective upon the impact of these disorders on the lives of these young patients and their families. This papers also aims to emphasize the fact that trauma can really affect one's emotional stability, and can be considered a trigger factor for different types of psychiatric conditions. Methods: We present the clinical case of a 17-year old female patient, hospitalized in emergency for psychomotor agitation, aggressive behavior, dromomania, autolytic ideation. Data on the clinical manifestation, trigger factors, treatment and evolution are discussed. Conclusions: When it comes to treating adolescents, whether it is depression, anxiety or other psychiatric disorders, treatment should be prescribed with caution, because the symptomatology is not very clearly defined. This polymorphism can offer a higher level of difficulty when it comes to establishing the right diagnosis.
\end{abstract}

\section{KEY WORDS:}

Depression, psychosis, manic episode, diagnosis criteria, differential diagnosis. 


\section{BACKGROUND}

Psychosis is a common and functionally disruptive symptom of many psychiatric, neurodevelopmental, neurologic, and medical conditions and an important target of evaluation and treatment in neurologic and psychiatric practice (1).

Psychosis is the defining feature of schizophrenia spectrum disorders, a common but variable feature of mood and substance use disorders, and a relatively common feature of many developmental, acquired, and degenerative neurologic and medical conditions. Across these conditions, psychosis is both a contributor to disability and a barrier to productivity and participation. Psychosis is, therefore, an important target of evaluation and treatment among patients receiving care from neurologists and psychiatrists. (2, 3, 4, 5)

Central to all of the presently available guidelines on the evaluation and management of patients with psychosis is the need to identify the cause of psychotic symptoms (in order of priority: delirium, including delirium due to substance intoxication/withdrawal; secondary psychoses of neurologic, medical, and substance use disorders; mood disorders with psychotic features; schizophrenia spectrum disorders; and other psychotic disorders) through comprehensive neuropsychiatric assessment. Patients should be involved in psychiatric, neurologic, and general medical history taking to the extent that their clinical status allows, and, whenever possible, collateral and corroborative history should be obtained from family members or others knowledgeable about the patient and from medical records. Evaluation for potentially causative or contributory medications (e.g., prodopaminergic or anticholinergic agents) and substance use disorders is essential. (6)
Evaluation for manic and depressive episodes (current and lifetime) anchored to DSM-5 criteria should be performed, as strict adherence to these criteria is required to distinguish between schizophrenia mood disorders with psychotic features and schizoaffective disorder. The DMS-5 encourages use of the Clinician-Rated Dimensions of Psychosis Symptom Severity scale to rate the severity of both manic and depressive symptoms associated with schizophrenia spectrum and other psychotic disorders.

Neuropsychological and occupational therapy consultations contribute usefully to cognitive and functional assessments and should be performed when feasible. (7)

The DSM-5 outlines the criteria to make diagnosis of Brief Psychotic Disorder.

Symptoms such as delusions, hallucinations, disorganized speech or catatonic behavior are sufficient. The duration of an episode should be at least 1 day but less than a month, with eventual full return to premorbid level of functioning.

The disturbance is not better explained by major depressive or bipolar disorder with psychotic features such as schizophrenia or catatonia, and is not attributable to the psychological effects of a substance. (e.g., a drug abuse, a medication) or another medical condition. There needs to be specified if the symptoms occur in response to events that singly or together, would be markedly stressful to almost anyone in similar circumstances in the individual's culture (brief reactive psychosis).

Major depression is a chronic illness with a high prevalence and is a major component of 
disease burden. Depressive disorders were the second leading cause of years lived with disability in 2010 in Canada, the United States and globally (Global burden of disease attributable to mental and substance use disorders: findings from the Global Burden of Disease Study 2010). (8)

When depression-related deaths due to suicide and stroke are considered, depression has the third highest global burden of disease. (9)

The DSM-5 outlines the criteria to make diagnosis of depression.

The individual must be experiencing five or more symptoms during the same 2-week period and represent a change from previous functioning; at least one of the symptoms is be either (1) depressed mood, or (2) loss of interest or pleasure.

Symptoms include: depressed mood most of the day, nearly every day, as indicated by either subjective report, or observation made by others. Markedly diminished interest or pleasure in all, or almost all activities most of the day, nearly every day. Significant weight loss, or decrease or increase in appetite nearly every day. Insomnia or hypersomnia nearly every day. Psychomotor agitation or retardation nearly every day (observable by others, not merely subjective feelings of restlessness or being slowed down). Fatigue or loss of energy nearly every day. Feelings of worthlessness or excessive or inappropriate guilt nearly every day. Diminished ability to think or concentrate or indecisiveness nearly every day. Recurrent thoughts of death, recurrent suicidal ideation without a specific plan or a suicide attempt.

These symptoms must cause clinically significant distress or impairment in social, occupational, or other important areas of functioning.
The episode must not be attributed to the psychological effects of a substance or to another medical condition.

Mania, or a manic phase, is a period of 1 week or more in which a person experiences a change in normal behavior that drastically affects their functioning. Mania is different from hypomania because hypomania does not cause a major deficit in social or occupational functioning, and it is a period of at least 4 days rather than 1 week. The defining characteristics of mania are increased talkativeness, rapid speech, decreased the need for sleep (unlike depression or anxiety in which the need for sleep exists, but there is an inability to sleep), racing thoughts, distractibility and psychomotor agitation. Some other hallmarks of mania are an elevated or expansive mood, mood lability, impulsivity, irritability, and grandiosity. If the individual experiencing these symptoms requires hospitalization, then this period automatically qualifies as true mania and not hypomania, even if the symptoms are present for less than one week. (10-11).

Many families bring their loved ones to the emergency room due to the excessive behavioral changes they have noticed over a brief period. Patients amid a manic phase commonly engage in goal-directed activities that may result in harmful consequences, such as spending excessive money, starting businesses unprepared, traveling, or promiscuity. Many patients engage in property damage or even harm themselves or others through verbal or physical assaults. They may also become highly aggressive, agitated, or irritable. Although the patient may have poor insight and may not recognize they are behaving out of the norm, it becomes apparent to family or friends that this behavior may be due to mental illness. (12) 


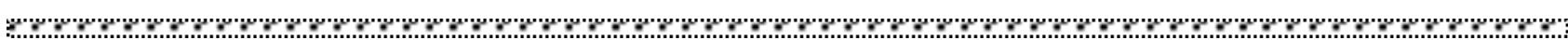

The DSM-5 outlines the criteria to make diagnosis of a Manic Episode

The patient presents an elevated, expansive or irritable mood and abnormally and persistently increased goal-directed activity or energy, lasting at least one week and present most of the day, nearly every day. During the period of mood disturbance and increased energy or activity, three or more of the following symptoms are present to a significant degree and represent a noticeable change from usual behavior: inflated selfesteem or grandiosity, decreased need for sleep, more talkative than usual or pressure to keep talking, flights of ideal or subjective experience that thoughts are racing, distractibility, excessive involvement in activities that have a high potential for painful consequences.

The mood disturbance is sufficiently severe to cause marked impairment in social or occupational functioning or to necessitate hospitalization to prevent harm to self or others, or there are psychotic features.

The episode is not attributable to the physiological effects of a substance (a drug abuse, a medication, other treatment) or to another medical condition.

A full manic episode that emerges during antidepressants treatment (e.g., medication, electroconvulsive therapy) but persists at a fully syndromal level beyond the physiological effect of that treatment is sufficient evidence for a manic episode and, therefore, a bipolar I diagnosis.

\section{CASE PRESENTATION}

Reasons for admission at the hospital:

A 17-year old female patient from Suceava presents for admission to the "Socola" Institute of Psychiatry, Iași, accompanied by her parents for sudden, onset of psychomotor restlessness, aggressive behavior, dromomania, autolytic ideation, emotional lability. False beliefs, inappropriate behavior, difficulties carrying out daily activities were also observed.

\section{PAST PERSONAL MEDICAL HISTORY AND FAMILY HISTORY}

The parents declare their daughter was diagnosed with depression a year prior following a traumatic event.

Family history- noncontributory.

\section{HISTORY OF PRESENT ILLNESS}

The family reports that a year prior, the patient was diagnosed with severe depression. The episode followed what the patient named "a traumatic event". The sister got married and moved to another country; the poor relationship with the brother in-law and the sister's departure have had a huge impact on the teenager. She was treated as an outpatient, the treatment consisting in antidepressants. She has received this treatment for a year.

The relationship with the parents is poor and the patient is accusing them of abuse of authority. She resents her father and she affirms that the relationship with her mother is superficial. She cares the most about her sister, and now she believes that she abandoned her. The autolytic ideation appeared following a set of events.

Parents affirm that during the month prior the hospitalization, the patient showed an aggressive behavior towards them, mostly verbal, lost interest in all kinds of activities, poor activity in school, disorganized behavior, weight loss, emotional lability. The patient refused psychological counselling. 
A month prior hospitalization, the patient has been involved in a car accident. No severe trauma has been detected. The patient affirms that the injuries she suffered were severe and had a huge impact on her well-being, affecting her mental health the most. The driver was her boyfriend at the time. After the accident, the boyfriend left the country for work. The patient refers to this episode as highly traumatizing and once again, she feels abandoned.

A few weeks after the accident, the patient visited a male friend. The patient affirms that this friend has abused her sexually, being held hostage. She affirms that the sexual act was not consented and that she was a rape victim. She did not call the police or her parents.

She was later on taken to the Emergency Room where no signs of violence have been detected. She kept affirming that she was raped, therefore a gynecological examination was required in order to establish if she was a victim of a sexual assault; no signs of sexual abuse were detected. A pregnancy test was also made. The result was negative.

They redirect the patient to our clinic and the next day she is admitted.

\section{THE PSYSICAL EXAM}

Did not lead to any pathological findings.

The Clinical Examination reveals a nervous female patient, a BP of 109/69 mmHg, HR of 71, rhythmic heart sounds, normal pulmonary auscultation, no abdominal pain on palpation.

An ECG was performed and it showed sinus rhythm and no signs of ischemia.

\section{THE PSYCHIATRIC EXAM}

\section{Appearance and General Behavior:}

Attitude: cooperative, aggressive behavior, curious, psychomotor restlessness, emotional lability.
Clothing: neat, good hygiene status.

Voice: accelerated verbal flow, emotionally modulated, voice of medium tonality and increased intensity.

Look: establishes and maintains visual contact with the examining physician.

Facies: mobile

Mimics and Pantomime: gestural activity slightly increased.

\section{Cognitive functions:}

Sensation: slight hyperaesthesia.

Perception: she denies the presence of hallucinatory phenomena.

Attention: spontaneous hyperprosexia, marked distraction.

Memory: selective evocation hypermnesia for events related to the patient's personal life.

Thinking unfolds in a disorganized, accelerated rhythm, presenting pressured speech, racing thoughts, delusions of grandiosity.

Imagination: without disturbances.

\section{Affective and motivational functions:}

Mood: elevated

Agressive behavior and negative emotions towards the family.

Feelings: inadequate.

Passions: insufficiently structured.

Motivation: periods of delay in initiating activities.

Instincts

- $\quad$ eating-diminished

- preservation- autolytic ideation

- sexual- exacerbated.

\section{Executive functions:}

Volition: high spontaneity but with low involvement in activites, excessive in goaldirected activities (she wants to leave the country and get two jobs; she also wants to finish her studies with the highest grades and study medicine). 
ryor

Motor activity: self conduct and self care abilities maintained.

Verbal activity: rapid, difficult to interrupt, pressured speech

Behavior: uninhibited, theatrical behavior which included dramatic mannerisms.

Sleep: decreased need for sleep.

\section{Judgement and Insight:}

Conscience: orientation in space, time, and person preserved.

Insight over illness: absent.

Intellect: in accordance with educational background.

Character: inappropriate attitude.

Psychological examination: manic configuration with elevated mood, accelerated thought process, instability traitsm integrative and relational deficiency.

She presents a theatrical attitude, demonstrative behavior, affirms that she was the victim of a sexual trauma (rape), emotional lability, low tolerance to minor frustration, irritability, irascibility.

POSITIVE DIAGNOSIS: Manic episode with psychotic symptoms.

\section{DIFFERENTIAL DIAGNOSIS}

1. Organic affective disorders (organic manic disorder); a month prior admission, a CT scan was performed. No abnormalities were observed. Following her admission, we eliminated somatic diseases and the consumption of drugs.

2. Psychotic disorders, primarly delusional- the symptoms of this disorder have an abrupt onset, with no prodromal phase, usually caused by a traumatic event and the symptoms are are connected thematically with the stressful situation; the patient's delusions are mainly of grandeur.

3. Psychotic disorders induced by certain life conditions or by substance abuseno substance abuse was detected.

4. Schizoytypal disorder: symptoms which meet the criteria for this disorder include ideas of reference, odd beliefs with mystical or paranormal influences, observations that could not be made in this patient's case.

5. Persistent delusional disorders: include a multitude of pathologies that are characterized by the presence of delusions for at least 3 months.

6. Antisocial personality disorder: this disorder is characterized by the lack of respect towards other people and other people's rights. The patient in incapable to adapt to social and legal norms.

7. Schizoaffective disorder: both affective and schizophrenic symptoms can be observed during the same episode. No schizophrenic symptoms can be observed in this case.

During hospitalization the patient was under strictly supervised treatment.

The patient was anxious, showed signs of irritability, claiming, "you are going to have to deal with me from now on!", theatrical behavior.

The following days, the patient's evolution was stationary. She maintained the aggressive behavior. She had elevated self-esteem, and kept threatening the medical staff "I am going to unleash hell on all of you", "Enough with being a good girl. I am going to become a devil", "I am not crazy, I am just acting crazy", "I declare war", "You are going to see what I am capable of".

The patient accused the doctor and her parents of abuse of power "I want you to know that 


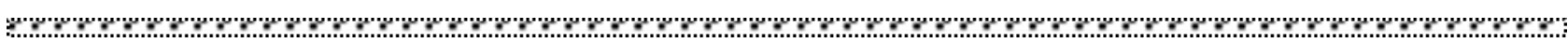

you have disappointed me, yes, you, the ones who were supposed to take care of me; you abandoned me in a mental institution! Why? Why am I always wrong? Set me free! It is only your fault! Lack of love!".

She insisted on the fact that she was pregnant following the "rape".

Another pregnancy-test was made. The result was negative. She then refused a gynecological examination.

After a few days of treatment, the treatment consisting in antipsychotics, the evolution was still stationary. The patient became demanding, she still presented aggressive behavior. "I am not crazy! I am just pregnant".

"Her mother's hope...cleaning tables dreaming about the day when she will wear a white robe and she will be walking down the halls of the hospital, bringing comfort to the patients". "May God be with you. Don't forget to talk to Him!".

A few days after continuing the treatment, the patient began to follow the rules. She insisted on helping the medical staff with all the chores and activities. She praised the doctor and the nurses. She told everyone she loved them. Her behavior suffered obvious adjustments. She was calm, cooperative, communicative, she showed no signs of aggressive behavior. "My family is perfect for me. Mum + Dad= Me + My sister", "I absolutely adore and love these people", "My dear parents, my love for you is infinite", "My family is loving, perfect", "I thank God every single day for my family", "My dear parents, please, I want you to understand! I want to come home! Please take me home! I am going to be how you want me to be; just take me home".

\section{DISCUSSIONS}

This case is illustrative for the evolution of psychiatric disorders in adolescents. After the first traumatic event, the patient was diagnosed with depression. She received treatment which consisted in antidepressants for a year. The symptomatology at the moment of admission indicated a psychotic episode, taking into consideration her false beliefs, inappropriate behavior and difficulties carrying out daily activities. The severity of this case is represented by the autolytic ideation and dromomania, the refusal of counselling or any type of social support. On her admission, antipsychotic was prescribed. Some of the symptoms disappeared, but, unfortunately, a shifting from psychosis to mania has been observed within a few days.

The patient had a decreased need for sleep, poor judgement, grandiose beliefs, disconnected thoughts, inappropriate irritability, pressure to keep writing to her parents, attention was easily drawn to unimportant and irrelevant items. Her speech was always rapid, difficult to interrupt, she presented a theatrical behavior which included dramatic mannerisms. Taking into consideration the relationship with her family, her speech was always marked by hostile comments and complaints. Flight of ideas was also observed.

The strong religious beliefs, the excessive authority found within the family environment, the supposedly abandonment involving her sister, the car accident, the departure of her boyfriend and the misunderstanding that she has come across for the past few months, severely affected the patient's emotional stability. She refers to these events as highly traumatizing. She resents her family, she believes that her situation was caused by the people who were supposed to take care of her and to offer support. No evidence of self-guilt was observed. 


\section{CONCLUSIONS}

We need to underline the importance of trauma, especially when it comes to adolescents. Trauma can really affect one's emotional stability and evolution. In this case, the patient's family was dysfunctional, she felt emotionally abused, she felt abandoned and misunderstood. The car accident can also be included on the list of stressors. Even if no evidence of a sexual assault exists, the patient is firmly convinced that she is a rape victim. This last event also influences the evolution, being considered by the patient as highly traumatizing.

When it comes to treating depression in adolescent patients, caution is required. Our case report suggests that the prescription of antidepressants to adolescents can be followed by severe complications, such as the shift to psychosis.

Emotional support is essential when it comes to treating depression. A safe and warm environment must be provided in order for the patient to feel comfort and understanding from the family members and society. Therefore, psychological counselling is highly recommended.

\section{ACKNOWLEDGEMENTS AND DISCLOSURES}

The authors declare that they have no potential conflicts of interest to disclosure.

\section{REFERENCES}

1. David B. Arciniegas, Psychosis, Continuum (Minneap Minn) 2015 Jun; 21 (3 Behavioral Neurology and Neuropsychiatry).

2. Morgan C, Lappin J, Heslin M, et al. Reappraising the long-term course and outcome of psychotic disorders: the AESOP-10 study. Psychol Med 2014; 44 (13): 2713-2726. doi:10.1017/S0033291714000282.

3. Rabinowitz J, Berardo CG, Bugarski-Kirola D, Marder S. Association of prominent positive and prominent negative symptoms and functional health, well-being, healthcare-related quality of life and family burden: a CATIE analysis. Schizophr Res 2013; 150 (2-3): 339-342. doi:10.1016/j.schres.2013.07.014.

4. Vilalta-Franch J, Lopez-Pousa S, Calvo-Perxas L, Garre-Olmo J. Psychosis of Alzheimer disease: prevalence, incidence, persistence, risk factors, and mortality. Am J Geriatr Psychiatry 2013; 21 (11): 1135- 1143. doi:10.1016/j.jagp.2013.01.051.

5. Forsaa EB, Larsen JP, Wentzel-Larsen T, et al. A 12-year population-based study of psychosis in Parkinson disease. Arch Neurol 2010; 67 (8): 996- 1001. doi:10.1001/archneurol.2010.166. Lehman AF, Lieberman JA, Dixon LB, et al. Practice guideline for the treatment of patients with schizophrenia, 2nd ed Arlington: American Psychiatric Association, 2004.

6. Clinician-rated dimensions of psychosis symptom severity. American Psychiatric Association. Published 2013. Accessed April 9, 2015.

7. Whiteford HA, Degenhardt L, Rehm J, Baxter AJ, Ferrari AJ, Erskine HE, Charlson FJ, Norman RE, Flaxman AD, Johns N, Burstein R, Murray CJ, Vos T

Lancet. 2013 Nov 9; 382(9904):1575-86.).

8. Ferrari AJ, Norman RE, Freedman G, et al. The burden attributable to mental and substance use disorders as risk factors for suicide: findings from the Global Burden of Disease Study 2010. PLoS ONE. 2014;9;e91936.

9. Wang YY, Xu DD, Feng Y, Chow IHI, Ng CH, Ungvari GS, Wang G, Xiang YT. Short versions of the 32-item Hypomania Checklist: A systematic review. Perspect Psychiatr Care. 2020 Jan;56(1):102-111. . Tazawa Y, Wada M, Mitsukura Y, Takamiya A, Kitazawa M, Yoshimura M, Mimura M, Kishimoto T. Actigraphy for evaluation of mood disorders: A systematic review and meta-analysis. J Affect Disord. 2019 Jun 15;253:257-269.

10. Kavanagh BE, Brennan-Olsen SL, Turner A, Dean OM, Berk M, Ashton MM, Koivumaa-Honkanen H, Williams LJ. Role of personality disorder in randomised controlled trials of pharmacological interventions for adults with mood disorders: a protocol for a systematic review and meta-analysis. BMJ Open. 2019 May 01;9(4):e025145.

\section{Correspondence:}

Elena-Rodica Popescu-M.D.

PhD Student, psychiatrist, "Socola” Institue of Psychiatry, Iași, România, no. 36 Str. Bucium, elenapopescu.88@yahoo.com 